\title{
Hand Vein Image Enhancement Based on Multi-Scale Top-Hat Transform
}

\author{
Guoqing Wang, Jun Wang, Ming Li, Yaguang Zheng, Kai Wang \\ School of Information and Electrical Engineering, China University of Mining and Technology, Xuzhou, \\ 221116 China \\ Emails: wangguoqingcumt@163.com_wj999lx@163.com 15062129100@163.com \\ wangguoqingatwork@163.com wangguoqingcumt@cumt.edu.cn
}

\begin{abstract}
Traditional image contrast enhancement methods originally cannot improve the quality of vein images and may also import some unknown noise resulting in low recognition rate. To overcome the abovementioned disadvantages, the paper proposes an enhancement method based on the morphological filtering theory including three main procedures. Firstly, the algorithm extract the vein Region Of Interest (ROI), and then adopting the improved White Top-Hat transform (WTH) and Black Top-Hat transform (BTH) methods to get the features of vein in detail in both white and black pattern (vein information and background information); Secondly, to construct the filtering function with the self-designed controlling operator, representing the gradient changes of the vein edges, which well reflects the importance of local detail in multi-scale pattern; Finally, traditional nonlinear gray-level transformation function is imported with modality to the parameters to realize the gray normalization. We perform rigorous experiments with the proposed method and other state-of-the-art enhancement methods on the self-built dorsal vein image databases, and the experimental results illustrate that the multiscale top-hat theory-based enhancement methods improve the contrast of hand vein images with restrictions on the possibility of enhancement on existing noise information.
\end{abstract}

Keywords: Contrast enhancement, morphological filtering, multi-scale, top-hat, vein recognition.

\section{Introduction}

Vein imaging methodology is based on the hand biometrical phenomenon where the hemoglobin, the main component of the venous blood, could well absorb the nearinfrared light at the wavelength of $760 \mathrm{~nm}$ or $850 \mathrm{~nm}$, while the other components are easily penetrated. Thus the vein images can be captured by CCD or CMOS with a certain set of near-infrared lights group reflecting on the hand or fingers. It can be 
inferred that vein information-based personalized identification has the advantages of high recognition rate and live body recognition $[1,2]$.

There have been at least three kinds of hand vein information-based biometric identification techniques including finger-vein recognition, palm-vein recognition and dorsal-vein recognition. The paper releases the outperformed enhancement methods focusing on the dorsal hand vein information which is easier to be captured. The proposed enhancement method is designed to decrease the influence resulted by the uncontrolled intensity of LED groups, and the specific influential situation could be described as: the stronger the intensity, the higher the global gray value with little visible vein information. On the other hand, the weaker the intensity, the poorer the contrast of the vein and background information. It can be inferred from the above disadvantages that designing a high-robust and well-performed enhancement method is necessary to obtain high recognition rate [3].

The paper proposes an enhancement method based on the morphological filtering theory to realize high-quality enhancement process, and it covers three main procedures: firstly, the algorithm extract the vein Region Of Interest (ROI), and then adopting the improved White Top-Hat transform (WTH) and Black Top-Hat transform (BTH) methods to get the features of vein detail in both white and black pattern (vein information and background information); Secondly, to construct the filtering function with the self-designed controlling operator representing the gradient changes of the vein edges from the perspective of effective control on the detailed vein information, which well reflects the importance of local detail in multi-scale pattern; Finally, traditional non-linear gray-level transformation function is imported with modality on the parameters to realize the gray normalization. In the evaluation part, the paper respectively design metrics from the perspective of quantization and visual effect, and we perform rigorous experiments with the proposed method and the state-of-the-art enhancement methods on the self-built dorsal vein image databases, and the experimental results illustrate that the multi-scale top-hat theorybased enhancement methods improve the quality and contrast of hand vein images with restrictions on the possibility of enhancement on existing noise information.

\section{Theory foundation}

\subsection{Top-hat transformation}

With the development of image structural elements designing methodology, the mathematical morphology theory-based image processing methods has become a more and more important branch of image processing tools with the advantages of locality, parallel computing, fast and easy to implement. The procedure of structural elements-based filtering is to collect the detailed topology distribution of vein image by treating the elements as probe going through every pixel of the image. And the resulted vein image could be obtained without non-vein information. The basic morphological process consists of four main formats including dilate, erosion, morphological open filtering and morphological close filtering, and the specific definition of them are as follows. 
Suppose that $f$ stands for the input image, $B$ stands for the designed structural element, the infinite erosion and dilation process on the gray image $f$ based on $B$ can be expressed as

$$
\begin{gathered}
f \Theta B=\min (f(x+u, y-v)-B(u, v)), \\
f \oplus B=\max (f(x-u, y-v)+B(u, v)) .
\end{gathered}
$$

Thus the corresponding open and close operation on $f$ could be expressed as

$$
\begin{aligned}
& f \circ B=(f \Theta B) \oplus B, \\
& f \cdot B=(f \oplus B) \Theta B .
\end{aligned}
$$

The definition of top-hat transform, which consists of WTH and BTH, can be figured out on the basis of the aforementioned open and close operation, the related expressions are

$$
\begin{aligned}
\operatorname{WTH}(x, y) & =f(x, y)-f \circ B(x, y), \\
\operatorname{BTH}(x, y) & =f \bullet B(x, y)-f(x, y) .
\end{aligned}
$$

It can be inferred from the definition of open and close operation that the open one can filter the "white-characteristic" pixels that are smaller than the pre-designed structural elements in size, while the closed one filters the dark characteristic pixels. Besides, it should be noted that the global gray level could be maintained during both the open and close operation. It can be concluded from that that the WTH transformation extracts the light character of the input image while the BTH transformation extracts the dark one.

\subsection{Multi-scale morphological filtering theory}

The intensive distribution of the image can be reflected on different scale levels due to the complicity and strong correlation among pixels for the image itself, especially for the image rich in texture characters such as the dorsal vein images. The vein detailed information would be ruined after the operation by the pre-defined structural elements at the same scale level, which is unavoidable, so the paper introduces the methods [3] of extracting the detailed character information at different scale level based on the defined multi-scale structural elements expressed as

$$
B_{n}=B_{0} \oplus B_{0} \cdots \oplus B_{0} \cdots, n-1 \text { times. }
$$

$B_{0}$ is the designed structural element, $n$ is the scale parameter implying that the high scale level element is dilated from the low level ones. The selected structural elements are isotropic and square-shaped to ensure the feature preservation after elements-based processing.

\section{Vein feature extraction based on the proposed multi-scale transformation}

\subsection{ROI extraction}

The rigorous enhancement experiment is performed on the self-built dorsal hand vein image database with a total of 500 images as shown in Fig. 1a, the ROI extraction is designed and placed at the first step to remove the background part and non-vein 
information, which can also improve the WTH operation effect, the paper designs the ROI process based on the methods in paper $[4,5]$.

The first step of ROI extraction is for the median filtering process to remove the noise, and then adopt the fixed threshold (the value is set as 0.5 based on the numerous of experiments statistical analysis) segmentation methods to realize the binarization on the image, the edge extraction is realized with the method "Sobel" after the noise removal and binarization process. The following geometry operation on the edge region involves finding and labeling the outer circles which are tangent to each other, the sequential process is to get the located angular points by judging the amount of intersection points between the circles and edge lines, and then getting the mid-perpendicular on the connection lines between the angular points for the purpose of figuring the perpendicular slope out. Finally, the ROI with the preset size of $180 \times 180$ could be obtained after the suitable rotation correction. The extraction process could also solve the problem of rotation and uneven illumination. The specific result of ROI extraction is shown as Fig. $1 b$.

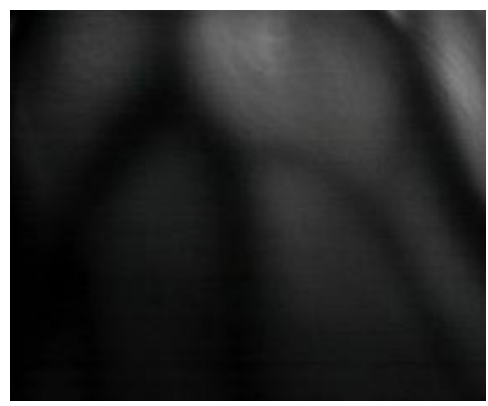

(a)

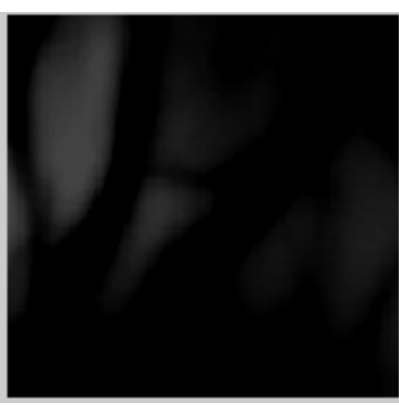

(b)

Fig. 1. Vein image samples: Original dorsal vein image (a); result of ROI process (b)

\subsection{Multi-scale feature representation}

S. Mukhopadhyay et al. [7], Bai, Zhou and Xue [8, 9] have designed the multi-scale top-hat transform for the image enhancement with the strategy of conducting enhancement on the detailed feature above different scale levels, but there exists great redundancy when realizing the enhancement operation and they are all lacking in flexibility with the unified criterion on the detailed feature, and the cited processing expressions are

$$
g=f+0.5 S_{\mathrm{o}}-0.5 S_{\mathrm{c}} .
$$

The calculating parameters are defined that:

$$
\left\{\begin{array}{l}
S_{\mathrm{o}}=\sum_{i=1}^{n}(n-i+1) \mathrm{WTH}_{i}, \\
S_{\mathrm{c}}=\sum_{i=1}^{n}(n-i+1) \mathrm{BTH}_{i} .
\end{array}\right.
$$

In the theory expressions, $f$ stands for the input image, $g$ stands for the enhanced image. It can be analyzed from this that the realization of enhancement is based on the repeated adding operation of the detailed features which results in lacking of 
operation adaptability. As mentioned above, the WTH transform can extract the light feature of images that is smaller than the structural elements in size while the BTH can extract the dark feature, so the idea of importing the control factor to make adjustment to the extracted detailed feature is fulfilled to realize the contrast enhancement by way of enlarging the gray value diversity. And the above theorybased process consists of ROI extraction and extracting the difference value at the adjacent scale which is able to adequately avoid the unnecessary enhancement to the detailed information. The feature extraction at different scale " $i$ " can be fulfilled by the next equation:

$$
\left\{\begin{array}{l}
\mathrm{WTH}_{i}=f-f \circ B, 0 \leq i \leq n, \\
\mathrm{BTH}_{i}=f \cdot B-f, 0 \leq i \leq n,
\end{array}\right.
$$

$\mathrm{WTH}_{i}, \mathrm{BTH}_{i}$ represent the light and dark detailed feature respectively, which are smaller than the preset structural elements.

The infinite feature extraction methods involve extracting the vein light and dark feature for later process, and the expressions to realize the extraction is fulfilled by

$$
\begin{gathered}
\left\{\begin{array}{c}
\mathrm{Xop}_{1}=f-f \circ B, \\
\mathrm{Xop}_{i}=\mathrm{WTH}_{i-1}-\mathrm{WTH}_{i}=f \circ B_{i-1}-f \circ B_{i}, \quad i=2, \cdots, n, \\
X c l_{1}=\mathrm{f} \cdot \mathrm{B}-f,
\end{array}\right. \\
\left\{\begin{aligned}
\mathrm{Xcl}_{i}=\mathrm{BTH}_{i-1}-\mathrm{BTH}_{i}=f \cdot B_{i}-f \cdot B_{i-1}, \quad i=2, \cdots, n .
\end{aligned}\right.
\end{gathered}
$$

Suppose that the maximum decomposition scale is set as $n$, the result after multiscale top-hat processing based on the above equations could be expressed as

$$
\begin{gathered}
\text { Xop } \rightarrow\left\{\mathrm{Xop}_{\mathrm{o} 1}, \mathrm{Xop}_{2}, \cdots, \mathrm{Xop}_{i} \cdots, \mathrm{Xop}_{n}\right\}, \\
\mathrm{Xcl} \rightarrow\left\{\mathrm{Xcl}_{c 1}, \mathrm{Xcl}_{2}, \cdots, \mathrm{Xcl}_{i} \cdots, \mathrm{Xcl}_{n}\right\} .
\end{gathered}
$$

$\mathrm{Xop}_{i}, \mathrm{Xcl}_{i}$ represent the set of detailed information at the adjacent scale, and the key design for the later enhancement process is how to effectively enhance the detailed information while avoiding importing fake vein information. The solution is to import the control factor $\delta$ regarding to the different detailed character at the different scale, the feature expressions after the process are shown as

(14) $\mathrm{Xop}=\left\{\delta_{\mathrm{o} 1} \mathrm{Xop}_{o 1}+\delta_{\mathrm{o} 2} \mathrm{Xop}_{2}+\cdots+\delta_{\mathrm{oi}} \mathrm{Xop}_{i}+\cdots+\delta_{\mathrm{on}} \mathrm{Xop}_{n}\right\}$,

$$
\mathrm{Xcl}=\left\{\delta_{\mathrm{c} 1} \mathrm{Xcl}_{\mathrm{O} 1}+\delta_{\mathrm{c} 2} \mathrm{Xcl}_{2}+\cdots+\delta_{\mathrm{c} i} \mathrm{Xcl}_{i}+\cdots+\delta_{\mathrm{c} n} \mathrm{Xcl}_{n}\right\} \text {. }
$$

\section{Realization of enhancement based on the extracted multi-scale feature}

According to the analysis on the character of vein images, it can be concluded that the detailed character is mainly reflected on the contrast value between the vein information and the background information, so the enhancement degree on both the white and black region needs to be designed separately. The degree of enhancement on both the black and white region is set as the same weight after trials and efforts. And then, the nonlinear transformation is designed to realize the global normalization, the specific flow chart of the proposed enhancement method is as shown in Fig. 2. 


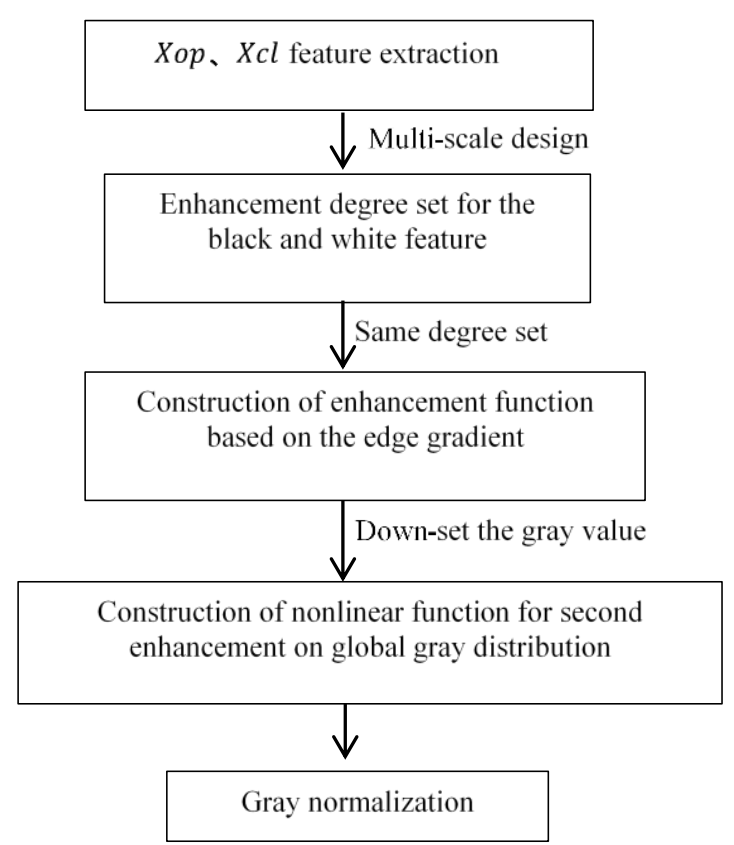

Fig. 2. Flow chart of the enhancement method based on the multi-scale top-hat transforms

4.1. Construction and experiment of enhancement function based on the edge gradient information

Based on the analysis on the feature distribution, it can be concluded that the white detailed feature information is seen as the background, while the corresponding black detailed one as the vein information, so the enhancement on the contrast is to strengthen the gray difference between the feature information and background information, the infinite transformation formulation is expressed as

$$
f_{\mathrm{e} n}=f+k \sum_{i=1}^{n} \delta_{i}\left(\mathrm{Xop}_{i}-\mathrm{Xcl}_{i}\right)
$$

where $k$ is set as 0.6 after numbers of experiments on the low quality vein images, $f_{\mathrm{e} n}$ is the expression of vein images after enhancement operation for the input image $f$. and the image edge gradient information is adopted in the design of the control factor $\delta_{i}$ for the reason that the later recognition experiment utilizes the edge information distribution orientation. The construction method of the infinite control factor $\delta_{\mathrm{i}}$ in every scale can be expressed as

$$
\delta_{i}(x, y)=\operatorname{Sigmoid}\left(h_{i}(x, y)-\frac{h_{i} \max +h_{i} \min }{2}\right) .
$$

The definition of $h_{i}$ is shown as follows:

$$
\begin{aligned}
& h_{i}=f \oplus B_{i}-f \Theta B_{i}, \\
& \operatorname{Sigmoid}(x)=\frac{1}{1+e^{\sigma x}} .
\end{aligned}
$$

Meanwhile, the $h_{i \max }$ and $h_{i \min }$ respectively stands for the maximum and minimum part of $h_{i}$. And the spatial distribution of the function relevant to the character of the vein image is a "big part in middle and gradually decrease towards the two sides from the middle". 


\subsection{Re-enhancement based on the constructed non-linear function}

According to the visual effect and feature parameters evaluation result we can conclude that the global gray value of the enhanced image is relatively high, so it is necessary to adjust the global gray value to improve the enhancement performance, where the paper introduces the nonlinear function for the re-enhancement process based on analysis results in paper $[9,10]$, the transformation is expressed as

$$
f_{\mathrm{e} 2}=G\left(f_{\mathrm{e}}(x, y)\right)=\left\{\begin{array}{cc}
2\left(f_{\mathrm{e}}(x, y)\right)^{2}, & 0 \leq f_{\mathrm{e}} \leq k, \\
1-2\left(1-f_{\mathrm{e}}(x, y)\right)^{2}, & k<f_{\mathrm{e}} \leq 1 .
\end{array}\right.
$$

In that expression, $k, k \in[0,1]$, is set so as the transform the turning point with the discipline so that when $f_{\mathrm{e}} \leq k$, the value is going nearer to 0 and when $k<f_{\mathrm{e}}$, the value is going nearer to 1 . And the $k$-value changing phenomenon is the result of function $G\left(f_{\mathrm{e}}(x, y)\right)$ and enhancement result changing with the value set, the final result is set as 0.5 after numerous experiments.

\subsection{Gray normalization}

After the process on the low quality vein image, the relatively high quality result is obtained with the unexpected result that the intensity distribution is more concentrated, which will bring the final recognition rate down, so it is necessary to import the normalization process which can strengthen the gray value region into $[0,255]$, the transformation is expressed as

$$
f_{\mathrm{e} n}=\frac{f_{\mathrm{e} 2}(x, y)-g_{\mathrm{min}}}{g_{\max }-g_{\min }} \times 255 .
$$

\section{Results and discussions}

The iterative image processing results by the described methods is as shown in Fig. 3.

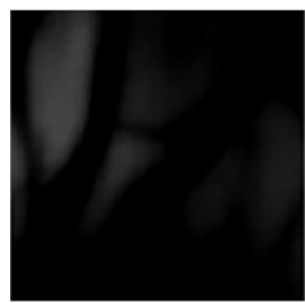

(a)

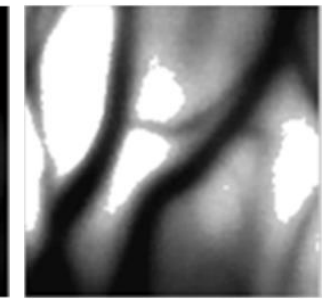

(b)

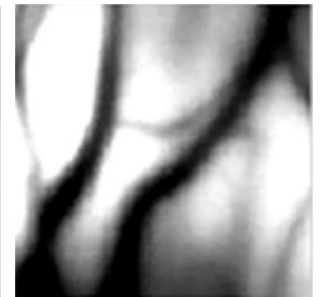

(c)

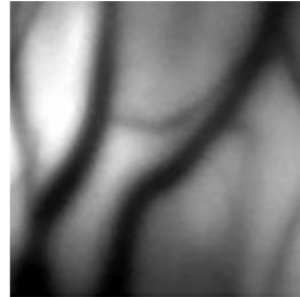

(d)

Fig. 3. Image processing result: Original vein image (a); enhancement by the function based on the edge gradient information (b); enhancement by the nonlinear function (c); gray normalization (d)

To demonstrate the effectiveness of the proposed method, the paper also introduces other state-of-the-art methods including HE (histogram equalization), Traditional Morphological Filtering method (TMF) are also introduced in the experiment design, The final evaluation system focusing on the visual observation is designed involving the quality evaluation parameter design and calculation of crosspoints. 
In the visual effect evaluation part, the enhancement part by HE has the disadvantages of high global value and local vein information distortion, resulting in loss of detailed vein information and low recognition rate; the result after TMF has the disadvantages of importing fake vein information with high redundancy, the proposed enhancement methods not only realize the enhancement on the feature information but also restrict the noise enlarging during the procedure which is of a great benefit for the later segmentation and recognition experiment, and it also brings about great improvement on the visual effect comparing to the introduced state-ofthe-art methods, the specific results are as Fig. 4.

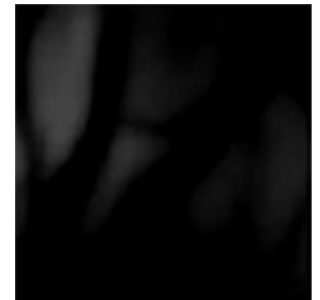

(a)

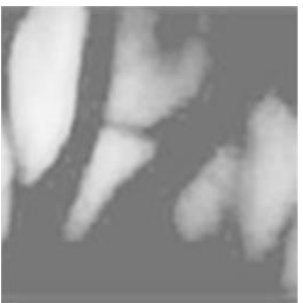

(b)

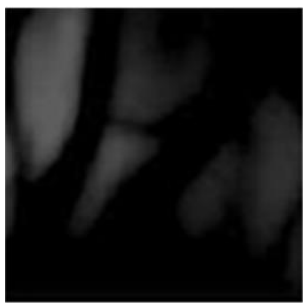

(c)

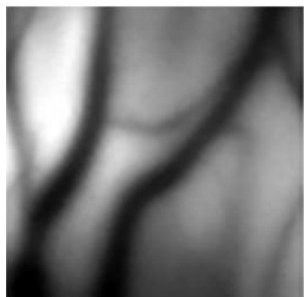

(d)

Fig. 4. Different enhancement processing results: Original vein image (a); HE (b); TMF (c); proposed method (d)

In the parameters evaluation part, the paper introduces the estimation including the calculation of information entropy and PSNR (Peak of Signal and Noise Ratio) to illustrate the statistical effect and the calculation of cross points before and after enhancement process respectively. And the corresponding calculation methods are expressed as

Information Entropy (IE)

$q(x)$ stands for the gray density after enhancement process;

PSNR evaluation

$$
\text { PSNR }=10 \lg \frac{255^{2} \times M \times N}{\sum_{i=1}^{M} \sum_{i=1}^{N}\left[f_{\mathrm{e}}(i, j)-f(i, j)\right]^{2}},
$$

$f$ and $f_{\mathrm{e}}$ respectively stands for the vein images before and after enhancement process, $M \times N$ is the size of the input image.

Meanwhile, the paper also introduces the linear fuzzy index $[11,12]$ to realize the evaluation on the image sharpness situation, where the infinite definition is

$f_{\max }$ is the maximum value of $f_{m}$.

$$
\begin{gathered}
\gamma\left(f_{\mathrm{e} n}\right)=\frac{2}{\mathrm{MN}} \sum_{x=1}^{M} \sum_{y=1}^{N} \min \left\{P_{x y} \cdot\left(1-P_{x y}\right)\right\}, \\
P_{x y}=\sin \left[\frac{\pi}{2}\left(1-\frac{f(x, y)}{f_{\max }}\right)\right],
\end{gathered}
$$

And it can be inferred from here that the higher the PSNR value, the better the enhancement effect with less noise creation, while on the other hand, the lower the $\gamma$ value, the higher the resolution. So the paper introduces the definition of descriptor PN (as 24 shows) that can well reflect the level of noise and sharpness of images.

$$
\mathrm{PN}=\frac{\mathrm{PSNR}}{\gamma} \text {. }
$$


Time consumption. To further compare the performance of the referred enhancement algorithms, the results of time consumption are also regarded as an important comparative factor. All the experiments have been run on the same X86_32 Windows PC with 2 GB of memory and i_ $3 \& 2+\mathrm{GHz}$ processor. It should be noted that the PC used for the experiment is relatively low-configuration to ensure convenient algorithm transplantation to embedded system for the design of portable vein recognition device. Besides, all the experiment is conducted with one sampled image sized as $180 \times 180$ pixels. The infinite result of the statistical parameters distribution is as shown in Table 1.

Table 1. Statistical comparison of different enhancement algorithms

\begin{tabular}{|c|c|c|c|c|c|}
\hline Algorithms & IE & PSNR & $\gamma$ & PN & Time, $s$ \\
\hline HE & 2.56 & 10.34 & 0.40 & 25.85 & 0.0523 \\
\hline TMF & 3.47 & 16.52 & 0.35 & 47.2 & 0.6489 \\
\hline Proposed & 3.85 & 21.54 & 0.42 & 51.29 & 0.6128 \\
\hline
\end{tabular}

It can be concluded from that that both the traditional evaluation (IE and PSNR) value and the newly-created PN value, obtained by the processed method, are higher than that of the traditional methods. The specific performance criterion is that the higher of IE, the more abundant of the detailed information; and the higher of PSNR, the better of image quality; the higher of PN, the more useful vein information and less noise creation.

As for the time consumption, it is far more satisfactory that the value of the proposed algorithm within 1s with extremely good enhancement result. However, it is 10 times of the classical HE model as a result of three-staged processing design: edge gradient information collection $(0.2659 \mathrm{~s})$, enhancement by the nonlinear function $(0.2137)$ and normalization procedure $(0.1332 \mathrm{~s})$. It is still more fastcomputation than the TMF model with a better enhancement performance.

\section{Conclusion and future work}

As for the research on vein recognition, the core problem is that the captured vein images cannot be used for feature extraction and recognition experiment directly, due to its low quality. Focusing on the problem, the paper proposes the enhancement algorithms combing the multi-scale top-hat transform with the special design of nonlinear function. Firstly, the algorithm constructs the edge gradient informationbased enhancement function for the first-layer process. In the second-layer process, the algorithm imports the design of the specific non-linear transformation function based on the first-layer result. Finally the algorithm imports the gray normalization process to strengthen the global gray distribution. The proposed algorithm, which can not only improves the detailed information distribution but also realizing the restriction in noise creation during filtering process, is fast-computation and betterperformance than the state-of-the-art enhancement methods.

For future extension of our work, we are going to work on designing an unsupervised method (i.e., k-means [13] and CNN [14]) to figure out the better control factor in edge gradient information calculation. What's more, direct enhancement on RGB images will be tried and discussed later to find another efficient 
framework on not only vein images but also other low-contrast medical images enhancement problem.

Acknowledgements: The research described in this paper has been supported by three main projects: Fundamental Research Funds for the Central Universities of China (Grant No 2011QNB21); Innovative engineering developing on graduates of Jiangsu Province in China (Grant No CXZZ11-0293); Chinese Coal Industry Association of science and technology guiding plan project (Grant No MTKJ2011-306); Innovative Training Project of the China university of mining and technology (Grant No XZD1029014185).

\section{References}

1. L i n, X., B. Z h u a n g, X. S u. Measurement and Matching of Human Vein Pattern Characteristics. - Tsinghua University (Science \& Technology), Vol. 43, 2003, No 2, pp. 164-167.

2. Z h a n g, J., M. S u n. Study on Algorithm for Skeleton Features Extraction of Hand Vein Image. Computer Applications, Vol. 27, 2007, No 1, pp. 152-154.

3. Lu, P., S. L a i. Image Enhancement and Feature Matching of Palm-Dorsa Vein. - Computer Engineering and Design, Vol. 28, 2007, No 5, pp. 3623-3625.

4. Suanta, M., C. Bhabatosh. A Multiscale Morphological Approach to Local Contrast Enhancement. - Signal Processing, Vol. 80, 2000, No 4, pp. 685-696.

5. K i m, M., M. K i m, K. L e e. Localization Method Using Vector Histogram. - IEEE Trans on Image Processing, Vol. 68, 2008, No 4, pp. 254-260.

6. J u n, W., G. W a n g et al. Hand Vein Recognition Based on Improved Template Matching. - Int. J. Bioautomation, Vol. 18, 2014, No 4, pp. 337-348.

7. S u nd ar a m, M., K. R a mar, N. Aru mu ga m et al. Histogram Modified Local Contrast Enhancement for Mammogram Images. - Applied Soft Computing, Vol. 15, 2011, No 2, pp. 5809-5816.

8. B a i, X., F. Zhou, B. X u e. Toggle and Top-Hat Based Morphological Contrast Operators. Computers \& Electrical Engineering, Vol. 38, 2012, No 5, pp. 1196-1204.

9. B a i, X., F. Z h o u, B. X u e. Image Enhancement Using Multi Scale Image Features Extracted by Top-Hat Transform. - Optics \& Laser Technology, Vol. 44, 2012, No 2, pp. 328-336.

10. T a r i k, A., Y. A $1 \mathrm{t} \mathrm{u} \mathrm{n} \mathrm{b} \mathrm{a} \mathrm{s} \mathrm{a} \mathrm{k.} \mathrm{Image} \mathrm{Local} \mathrm{Contrast} \mathrm{Enhancement} \mathrm{Using} \mathrm{Adaptive} \mathrm{Non-Linear}$ Filter. - In: Proc. of International Conference on Image Processing, 2006, pp. 2881-2884.

11. B h a b a to sh, C. Morphological Algorithm for Image Processing. - IETE Technical Review, Vol. 16, 2008, No 25, pp. 9-17.

12. I s h it a, D., C., B h a b a t o sh, B. Ch a t t o p a d h y a y. Enhancing Effective Depth-of-Field by Image Fusion Using Mathematical Morphology. -Image and Vision Computing, Vol. 24, 2006, No 3, pp. 1278-1287.

13. C o at e s, A., A. Y. N g. Learning Feature Representation with K-Means. - Neural Networks: Tricks of the Trade, 2012, 7700, pp. 561-580.

14. B a i 11 y, K., M. M i $1 \mathrm{~g} \mathrm{r}$ a m. Boosting Feature Selection for Neural Network Based Regression. Neural Networks the Official Journal of the International Neural Network Society, Vol. 22, 2009, No 5-6, pp. 748-756. 